\title{
Sensor Concept for Controlled Laser Cleaning via Photodiode
}

\author{
M. Lentjes, D. Klomp and K. Dickmann \\ Lasercenter Fachhochschule Münster (LFM), FB Physikalische Technik, \\ University of Applied Sciences, Stegerwaldstr. 39, 48565 Steinfurt, Germany \\ m_lentjes@fh-muenster.de
}

\begin{abstract}
In the field of laser cleaning of artworks the effect of "over-cleaning" is a commonly well known problem. The detection of laser induced plasma is one possibility in order to identify the kind of material just being irradiated by the laser beam. LIBS is a powerful method for the extraction of spectral information. Instead the detection of the plasma intensity contains much less information. However, this can be realised by using a fast photodiode. It has turned out that for several applications in laser cleaning of artworks a reliable identification of layers during the cleaning process is possible. In cooperation with restorers we proved that this low-cost method may be used for online monitoring as well as automated closed loop cleaning.
\end{abstract}

\section{Introduction}

In general laser removal of various layers from artworks takes place at high intensities of some $10 \mathrm{MW} / \mathrm{cm}^{2}$ accompanied by a plasma plume above the irradiated area. The properties of the plasma mainly depend on the laser intensity, laser wavelength and chemical composition of the ablated matter. The plasma signal is characterized by the duration, spectrum and amplitude.

Utilisation of the plasma signal for online process control in order to avoid over-cleaning is well known for laser cleaning of artworks [1-3]. Therefore

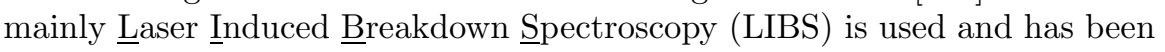
proved in several application cases [4-6]. In contrast to the spectrum of the plasma its integral value contains much less information about the removed matter. However, it may be sufficient for selected applications as has been reported in [7]. In our experimental setup we use a fast photodiode for the plasma detection. Furthermore in combination with separate optical filters even limited spectral information may be extracted from the plasma signal.

\section{Experimental Setup}

The schematic of the detection principle is shown in Fig. 1 For irradiation of various artwork samples we used a KrF-excimer laser (Lambda, LPX 305i) with maximum pulse energy of $1200 \mathrm{~mJ}$, repetition rate $1-50 \mathrm{~Hz}$, pulse duration $20-40 \mathrm{~ns}$ (@ $\lambda=248 \mathrm{~nm}$ ). A part of the laser induced plasma radiation 


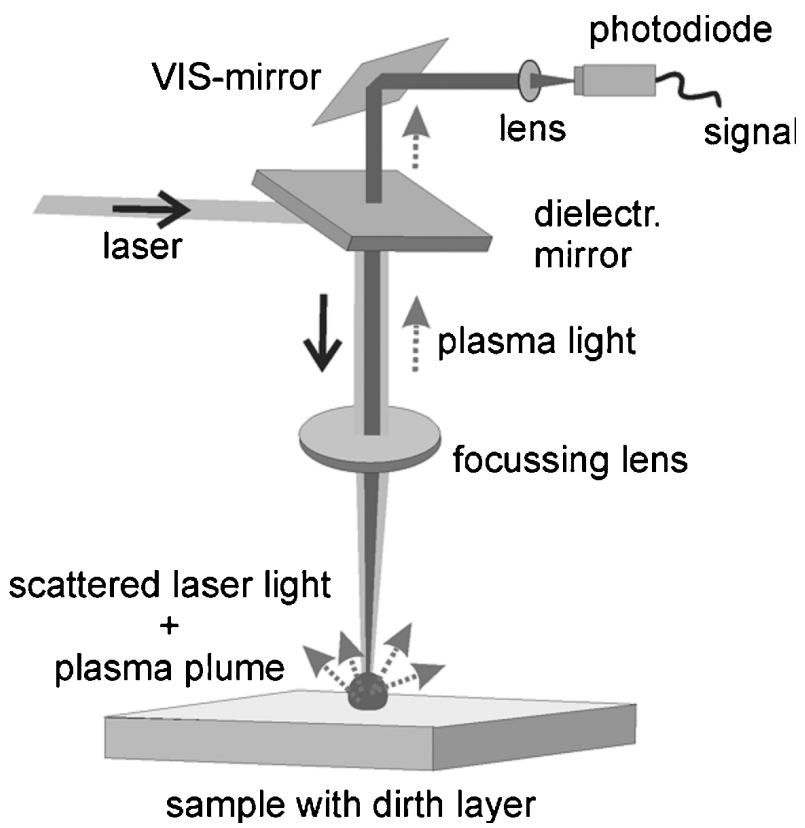

Fig. 1. Schematic diagram of the optical setup

is collimated by the focussing lens and is directed through the dielectric mirror (HR@248 nm) onto a fast silicon PIN photodiode ( $\left.\mathrm{T}_{\text {rise }}<1 \mathrm{~ns}\right)$. In the present case a separation of the scattered laser radiation from plasma radiation was ensured by the absorption of optical elements (@ $248 \mathrm{~nm}$ ) in front of the photodiode as well as low sensitivity of the photodiode @ $248 \mathrm{~nm}$.

The output signal of the photodiode may be used either for monitoring the cleaning process by the restorer or for automated closed loop cleaning. The corresponding setup is shown in Fig. 2. The oscilloscope, laser and xystage controller are linked up by a PC, which controls the data transfer and communication between these modules by a LabView written program.

\section{Control Process}

A reference value of the plasma signal ("compare level") being necessary for comparison of the required and actual signal has to be defined by the operator. This can be found either based on restorers experience or by a teaching procedure on test samples. During the laser removal process, the plasma signal of each laser pulse is compared with the reference signal. Laser removal will continue until the reference signal is reached. In a next step, corresponding to the spot size, the xy-control moves the sample to a new position directly beside it. The flow diagram is given in Fig. 3. 


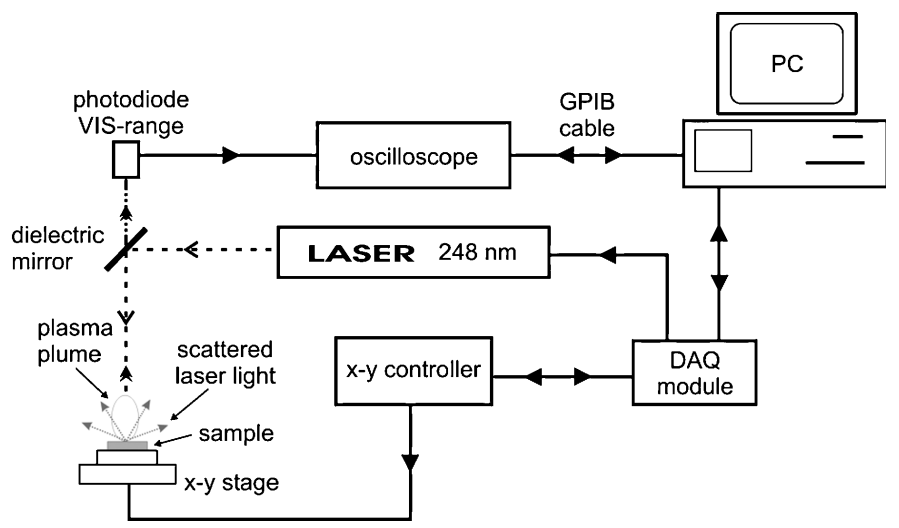

Fig. 2. Schematic of electronic setup for controlled laser cleaning via photodiode

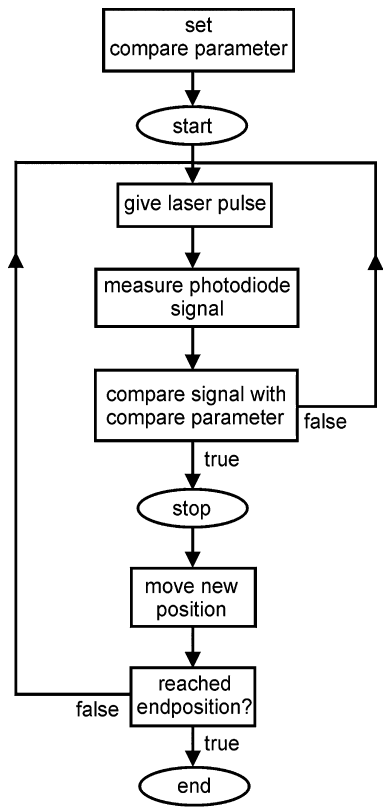

Fig. 3. Flow-diagram of closed loop cleaning operation

\section{Results}

On various layers/substrate-arrangements we observed a distinct alteration of the plasma signal going along with the removal process. Using this signal, not only different types of layers could be detected, but also gradients of compositions within the same layer. Figures $4 \mathrm{a}-4 \mathrm{~d}$ shows the recorded diode signals versus pulse numbers and the corresponding samples with cleaned areas. 


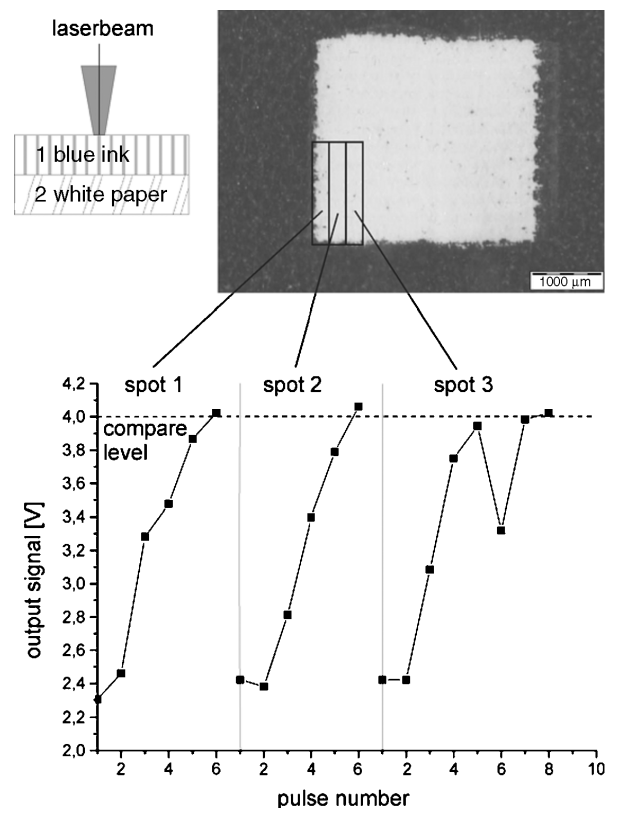

Fig. 4a. Blue ink removed from paper by controlled laser cleaning. Monitored photodiode signal (peak) vs number of laser pulses for the first three spots

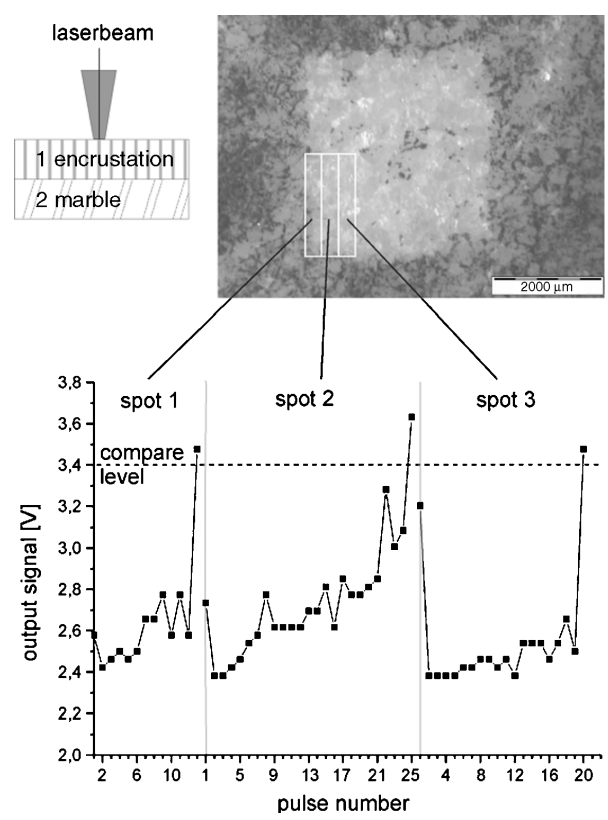

Fig. 4b. Encrustation removed from marble by controlled laser cleaning. Monitored photodiode signal (peak) vs number of laser pulses for the first three spots 


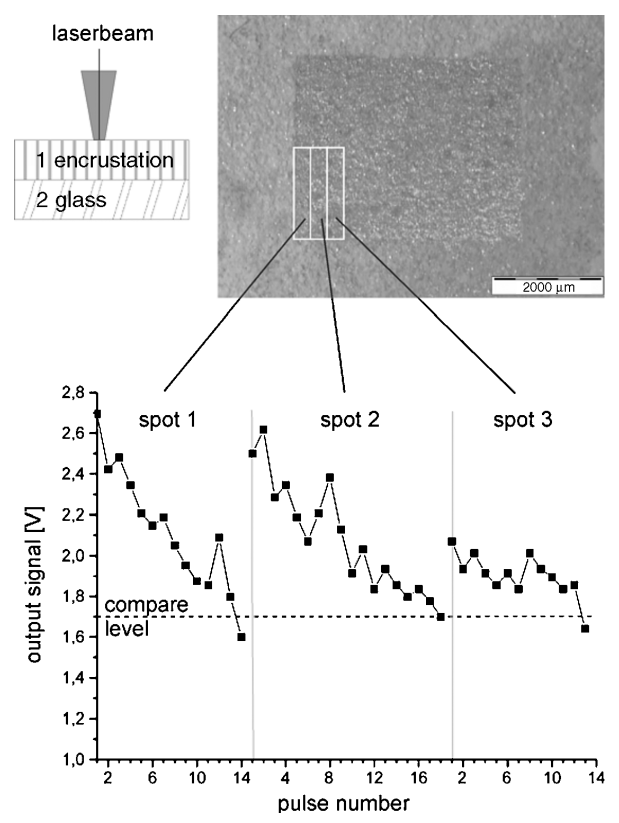

Fig. 4c. Encrustation removed from glass by controlled laser cleaning. Monitored photodiode signal (peak) vs number of laser pulses for the first three spots

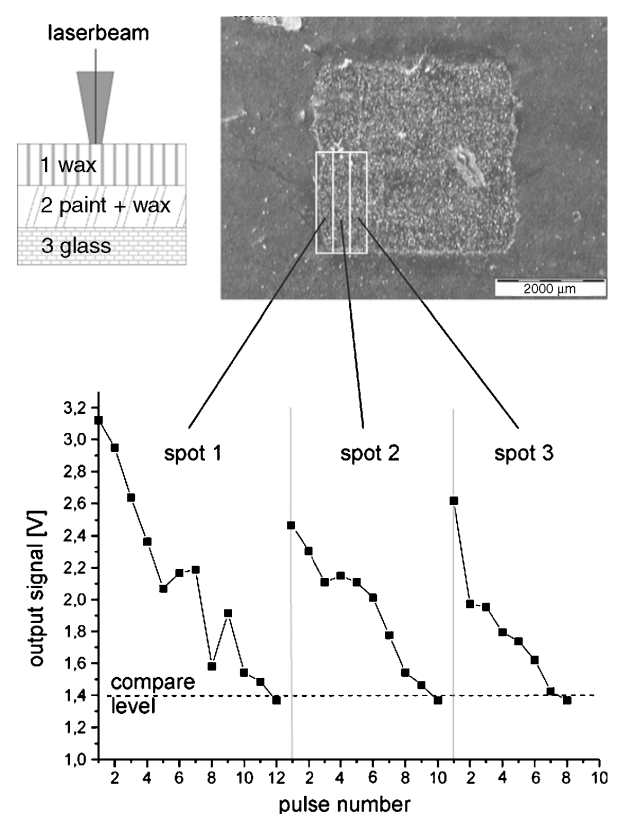

Fig. 4d. Wax-layer removed from paint by controlled laser cleaning. Monitored photodiode signal (peak) vs number of laser pulses for the first three spots 
In Figs. 4a and 4b there is a rise of the photodiode signal with increasing pulse number. In this case the plasma intensity is increasing when approaching to the original surface. The fast increasing graph in Fig. 4a shows that the composition within the same layer (here blue ink) differs, while penetrating deeper into this layer. In contrast, Fig. 4b reveals a more homogeneous composition of the layer to be removed. Fig. 4c and $4 \mathrm{~d}$ display a decreasing plasma intensity during the cleaning process. In these cases the distinct downward tendency of the detected plasma signals enables a reliable identification of various layers during the ablation process. Concluding, deduced from the Figs. 4a, 4b, 4c, 4d it is obvious that each individual spot on the surface needs a specific number of pulses for complete cleaning. With increasing inhomogeneity of the surface layer (e.g. artworks) this fact gains of importance. In first attempts we have used this detector principle for successful closed-loop laser cleaning.

\section{Acknowledgements}

This project is funded by the EUREGIO (INTERREG III Program) within the "EUREGIO CENTER for Art Restoration Technology" (ECEACT).

\section{References}

1. Gobernado-Mitre, A. C. Prieto, V. Zafiropulos, Y. Spetsidou, and C. Fotakis, On-Line Monitoring of Laser Cleaning of Limestone by Laser-Induced Breakdown Spectroscopy and Laser-Induced Fluorescence, in Appl. Spectroscopy, Vol 51, No 8, 1997, pp 1125-1129

2. P. V. Maravelaki, V. Zafiropulos, V. Kilikoglou, M. Kalaitzaki, and C. Fotakis, Laser-induced breakdown spectroscopy as a diagnostic technique for the laser cleaning of marble, in Spectrochimica Acta, Part B, 52, 1997, pp 41-53

3. D. Anglos, S. Couris, A. Mavromanolakis, I. Zergioti, M. Solomidou, W. Q. Liu, T. G. Papazoglou, V. Zafiropulos, C. Fotakis, M. Doulgeridis, and A. Fostiridou, Laser Induced Breakdown Spectroscopy (LIBS) and Laser Induced Fluorescence (LIF) Spectroscopy, LACONA 1, in Restauratorenblätter, Wien 1997, pp 113-118

4. S. Klein, T. Stratoudaki, V. Zafiropulos, J. Hildenhagen, K. Dickmann, and Th. Lehmkuhl, Laser-induced breakdown spectroscopy for on-line control of laser cleaning of sandstone and stained glass, in Appl. Physics A, 69, 1999, pp 441-444

5. J. H. Scholten, J. M. Teule, V. Zafiropulos, and R. M. A. Heeren, Controlled laser cleaning of painted artworks using accurate beam manipulation and on-line LIBS-detection, LACONA 3, in Journal of Cultural Heritage, Vol.1, Sup.1, 2000, pp 215-220

6. R. Teule, H. Scholten, O. F. v/d Brink, R. M. A. Heeren, V. Zafiropulos, R. Hesterman, M. Castillejo, M. Martín, U. Ullenius, I. Larsson, F. Guerra-Librero, A. Silva, H. Gouveia, and M. B. Albuquerque, Controlled UV laser cleaning of 
painted artworks: a systematic effect study on egg tempera paint samples, LACONA 4, in Journal of Cultural Heritage, Vol.4, Sup.1, 2003, pp 209-215

7. J. Hildenhagen and K. Dickmann, Low-cost sensor system for online monitoring during laser cleaning, LACONA 4, in Journal of Cultural Heritage, Vol.4, Sup.1, 2003, pp 343-346 